\title{
Hollandites as a new class of multiferroics
}

SUBJECT AREAS:

FERROELECTRICS AND MULTIFERROICS

MAGNETIC MATERIALS

SOLID-STATE CHEMISTRY

CHARACTERIZATION AND ANALYTICAL

TECHNIQUES

Received

19 June 2014

Accepted

7 August 2014

Published

27 August 2014

Correspondence and requests for materials should be addressed to

S.O. (sobrien@ccny. cuny.edu)
Shuangyi Liu'1,10, Andrew R. Akbashev ${ }^{2}$, Xiaohao Yang ${ }^{3}$, Xiaohua Liu', Wanlu Li', Lukas Zhao ${ }^{4}$, Xue Li ${ }^{5}$, Alexander Couzis ${ }^{5,10}$, Myung-Geun Han ${ }^{6}$, Yimei Zhu ${ }^{6}$, Lia Krusin-Elbaum ${ }^{4}$, Jackie Li ${ }^{7}$, Limin Huang ${ }^{1,8}$, Simon J. L. Billinge ${ }^{3,6}$, Jonathan E. Spanier ${ }^{2,9}$ \& Stephen $\mathrm{O}^{\prime}$ Brien $^{1,10}$

${ }^{1}$ Department of Chemistry, The City College of New York, Marshak Building, 160 Convent Avenue, NY 10031 , USA, ${ }^{2}$ Department of Materials Science \& Engineering, Drexel University, 3141 Chestnut Street, Philadelphia, PA 19104, USA, ${ }^{3}$ Department of Applied Physics and Applied Mathematics, The Fu Foundation School of Engineering and Applied Science, Columbia University, Mudd Building, 500 West 120 th Street, New York, NY 10027, USA, ${ }^{4}$ Department of Physics, The City College of New York, Marshak Building, 160 Convent Avenue, NY 10031, USA, ${ }^{5}$ Department of Chemical Engineering, The Grove School of Engineering, Steinman Hall, 160 Convent Avenue, The City College of New York, New York, NY 1003 1, USA, ${ }^{6}$ Condensed Matter Physics and Materials Science Department, Brookhaven National Laboratory, Upton, NY 1 1973, USA, ${ }^{7}$ Department of Mechanical Engineering, The Grove School of Engineering, Steinman Hall, 160 Convent Avenue, The City College of New York, New York, NY 10031 , USA, ${ }^{8}$ Department of Chemistry, South University of Science and Technology of China, Shenzhen 518500, P. R. China, ${ }^{9}$ AJ Drexel Institute for Energy \& the Environment, Drexel University, 3141 Chestnut Street, Philadelphia, PA 19104, USA, ${ }^{10}$ The CUNY Energy Institute, City University of New York, Steinman Hall, 160 Convent Avenue, The City College of New York, New York, NY 10031 , USA.

Discovery of new complex oxides that exhibit both magnetic and ferroelectric properties is of great interest for the design of functional magnetoelectrics, in which research is driven by the technologically exciting prospect of controlling charges by magnetic fields and spins by applied voltages, for sensors, 4-state logic, and spintronics. Motivated by the notion of a tool-kit for complex oxide design, we developed a chemical synthesis strategy for single-phase multifunctional lattices. Here, we introduce a new class of multiferroic hollandite $\mathrm{Ba}-\mathrm{Mn}$-Ti oxides not apparent in nature. $\mathrm{BaMn}_{3} \mathrm{Ti}_{4} \mathrm{O}_{14.25}$, designated $\mathrm{BMT}-134$, possesses the signature channel-like hollandite structure, contains $\mathrm{Mn}^{4+}$ and $\mathrm{Mn}^{3+}$ in a $1: 1$ ratio, exhibits an antiferromagnetic phase transition $\left(T_{N} \sim 120 \mathrm{~K}\right)$ with a weak ferromagnetic ordering at lower temperatures, ferroelectricity, a giant dielectric constant at low frequency and a stable intrinsic dielectric constant of $\sim 200$ (1-100 MHz). With evidence of correlated antiferromagnetic and ferroelectric order, the findings point to an unexplored family of structures belonging to the hollandite supergroup with multifunctional properties, and high potential for developing new magnetoelectric materials.

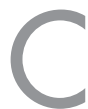

omplex oxides have been of long-standing interest due to the extensive range of properties derived from their electronic structure, and the library of metallic ions that can be intersubstituted in order to tune or transform the electronic behavior ${ }^{1-14}$. Pursuing single phase multiferroic magnetoelectrics ${ }^{15}$, uncommonly rare in high symmetry systems $s^{3,16,17}$, provides strong motivation to push further for synthetic exploration and rational design ${ }^{15,18}$. In addition to the fascintating science that has emerged, the goal is to search for stronger coupling between polarization $(P)$ and magnetization $(M)$, closer to room temperature, for device applications in memory (FRAM, MRAM) ${ }^{3}$, magnetic tunnel junction $\mathrm{s}^{19,20}$ and electric field induced behavior ${ }^{21}$. Progress has been partly limited by physical restrictions on the co-existence of substantive magnetic or electrical polarization in a single compound ${ }^{1,3}$. Composite materials can produce magnetoelectric effects through strain-mediated coup$\operatorname{ling}^{22-25}$, and the reliance on emergent phenomena at interfaces ${ }^{5,26}$, but the prospect of intimate coupling in a single crystal phase remains of great interest, since it relies on fundamental quantum mechanical interactions. In recent years the investigation of single phase multiferroics has grown to explore, both theoretically and experimentally, the means for placing lower constraints on the coexistence of ferroelectricity with ferromagnetism ${ }^{2}$. The results continue to ignite the potential for finding field tunable magnetoelectric behavior in novel compounds. Approaches have included the study of magnetically ordered improper ferroelectrics ${ }^{27-29}$, copper oxide ${ }^{30,31}$, mixed lead-iron perovskites ${ }^{32,33}$, Aurivilius-phase manganites and cobaltites ${ }^{34,35}$, and hexaferrites ${ }^{13}$.

We targeted synthesis of complex manganese and titanium oxide frameworks since they present possibilities for achieving direct spin-polarization coupling due to the diversity in magnetism of the manganese cations $(S=$ 5/2, 2 and 3/2 for $\mathrm{Mn}^{2+}, \mathrm{Mn}^{3+}$ and $\mathrm{M}^{4+}$ respectively), combined with the " $d^{0}$-ness" of $\mathrm{Ti}^{4+}$ cations, which can facilitate long range ordering through LUMO hybridization with $\mathrm{O} 2 \mathrm{p}$ orbitals, and drive bulk electric polariza$\operatorname{tion}^{16}$. In nature, and excluding silicates, there are fourteen oxides that contain $\mathrm{Ba}, \mathrm{Ti}$, and $\mathrm{O}$ ions, and fifteen that contain $\mathrm{Ba}, \mathrm{Mn}$ and $\mathrm{O}$ ions (co-residing with a number of other metal cations such as $\mathrm{Fe}, \mathrm{Cr}, \mathrm{V}$ etc.). Synthetic 

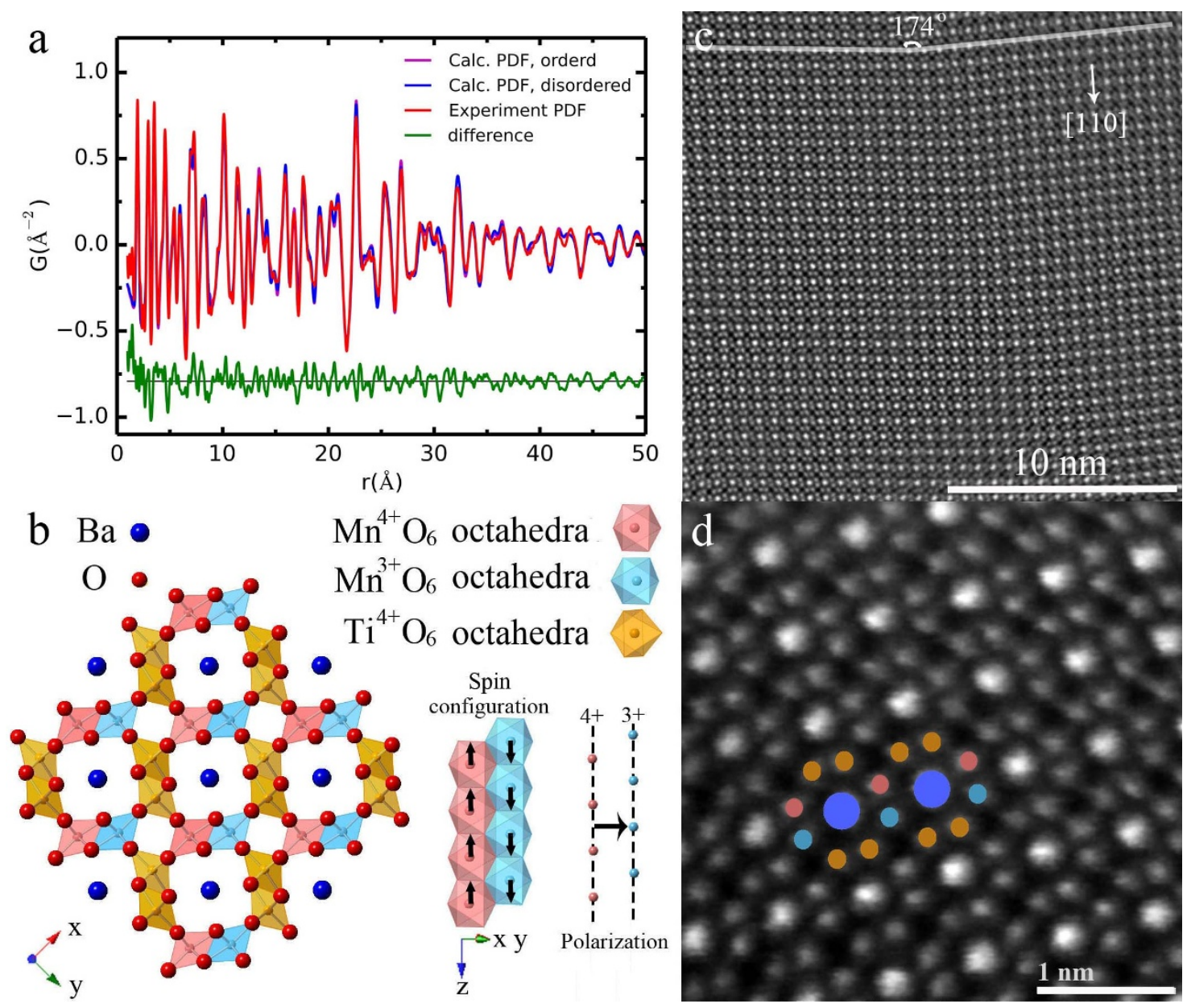

Figure 1 Structural characterization of BMT-134 ( $\left.\mathrm{BaMn}_{3} \mathrm{Ti}_{4} \mathrm{O}_{14.25}\right)$ (a), Synchrotron X-ray powder diffraction and PDF refinement patterns (b), crystal structure of BMT-134 projected down the $c$-axis, and a simple cartoon depiction of how spin and charge distribution in the lattice can cause collective polarization. (c) and (d), the atomic images obtained by spherical-aberration corrected STEM, with the zone axis along [001].

$\mathrm{Ba}(\mathrm{Mn}, \mathrm{Ti}) \mathrm{O}_{3}$ perovskites and polytypes have been prepared in the $1200-1600 \mathrm{~K}$ range to prepare high-permittivity compounds ${ }^{36-39}$, but surprisingly, there are no natural minerals classified as being composed of exclusively $\mathrm{Ba}, \mathrm{Ti}, \mathrm{Mn}$ and $\mathrm{O}$ ions, despite the fact that these are, respectively, the $14^{\text {th }}, 12^{\text {th }}$ and $9^{\text {th }}$ most abundant metals in the Earth's crust. A potential candidate crystal structure, where all these four elements may reside, is a hollandite supergroup, with a general formula of $\mathrm{A}^{\mathrm{II}}\left[\mathrm{M}^{\mathrm{IV}}, \mathrm{M}^{\mathrm{III}}\right]_{8} \mathrm{O}_{16}$, which comprises a number of mineral groups, including redledgeite. Redledgeite is a rare mineral that contains $\mathrm{Ti}$, with the formula $\mathrm{BaTi}_{6} \mathrm{Cr}_{2} \mathrm{O}_{16}{ }^{40}$; hollandite is a mineral that contains $\mathrm{Mn}$, with the formula $\mathrm{BaMn}_{8} \mathrm{O}_{16}{ }^{41}$. Thus, in principle, there are no fundamental chemical limitations to the formation of the $\mathrm{Ba}[\mathrm{Mn}, \mathrm{Ti}]_{8} \mathrm{O}_{16}$ hollandite.

Low temperature chemical synthesis and deposition methods for preparing high quality inorganic oxide films and nanostructures, especially ones that can be generalized ${ }^{42}$, are interesting for miniaturization and integration, to serve the needs of the electronics industry. Of particular interest is the use of atomic-scale building blocks that can mimic the precision of epitaxial growth using physical vapor deposition, and stimulate ordering within the lattice. The chemical synthesis method we employ here is a precursor driven technique derived from a combination of sol-gel ${ }^{43}$, non-aqueous ${ }^{44}$ and nucleation/growth-mediated nanocrystal synthesis ${ }^{45}$. The term "solution-processed" is now widely used to cover novel techniques that advance chemical alternatives to vacuum based physical deposition approaches. Our procedure is a solution processing technique described as gel-collection, due to the formation, collection and subsequent processing of a gel of nanocrystalline matter formed from the precursors with a preset stoichiometry (see Methods and Supplementary Information). We applied gel-collection to the synthesis of compounds of Ba-Mn-Ti-O. The first novel candidate structure of notable interest, $\mathrm{BaMn}_{3} \mathrm{Ti}_{4} \mathrm{O}_{14.25}$, is abbreviated to BMT-134.

\section{Results and Discussion}

The structure of BMT-134 was solved by a combination of synchrotron X-ray diffraction, high resolution transmission electron microscopy (HRTEM) and X-ray photoelectron spectroscopy (XPS). The crystal structure belongs to the hollandite supergroup, $\mathrm{A}^{\mathrm{II}}\left[\mathrm{M}^{\mathrm{IV}}\right.$, $\left.\mathrm{M}^{\mathrm{III}}\right]_{8} \mathrm{O}_{16}$. In this instance the fractional oxygen of the unit cell balances the $\mathrm{Mn}^{\mathrm{IV}}: \mathrm{Mn}^{\mathrm{III}} 1: 1$ ratio. X-ray powder diffraction data was analyzed using atomic pair distribution function analysis, $\mathrm{PDF}^{46-48}$ (Fig. 1a and Supplementary Information). The refinement result closely resembles the redledgeite structure $(I 4 / \mathrm{m})$. The $\mathrm{Mn}$ and $\mathrm{Ti}$ cations are located inside corner- and edge-shared oxygen octahedra with $\mathrm{Ba}$ cations in the channels (Fig. 1b). The Ba atoms have local displacements along the $z$-axis, with a fraction sitting off their equilibrium sites, identifiable at low $-r$ range $(<20 \AA)$. The sphericalaberration corrected STEM image shows the hollandite-type lattice, projecting down the channels (Fig. 1c, 1d). At lower magnification there is evidence of a small $\left(<6^{\circ}\right)$ lattice rotation, attributed to possible local cation disorder, but not twinning. XPS confirms the presence of stable $\mathrm{Ti}^{4+}$ and mixed $\mathrm{Mn}^{3+}$ and $\mathrm{Mn}^{4+}$ cations (Fig. 2a). No $\mathrm{Ti}^{3+}$ or $\mathrm{Mn}^{2+}$ is detected. The PDF refinement, EDX, EELS and XPS analyses all corroborate the $\mathrm{Ba}: \mathrm{Mn}$ : Ti stoichiometric ratio to be $1: 3: 4$, identical to the initial reactant concentration. 

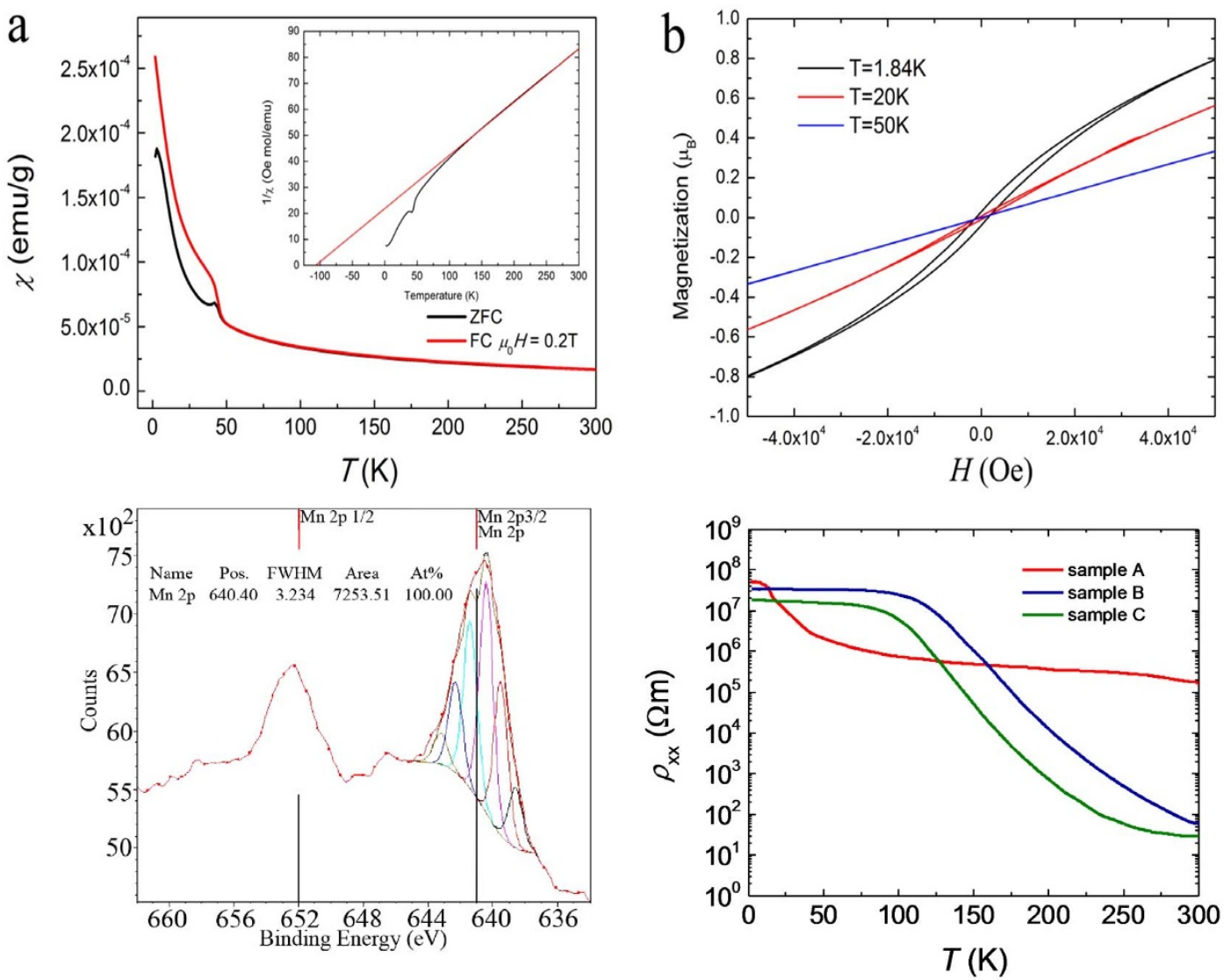

Figure 2 Magnetic and electronic transport properties, and electronic structure of BMT-134. (a), Temperature dependent magnetic susceptibility measured at $2 \mathrm{kOe}$ under FC and ZFC conditions. The inset shows the inverse ZFC susceptibility with a Curie-Weiss fit; (b), magnetic hysteresis loops at $50 \mathrm{~K}, 20 \mathrm{~K}$, and $1.84 \mathrm{~K}$. Deviation from linearity at $T_{\mathrm{N}} \sim 120 \mathrm{~K}$ and a local minimum at $T \sim 42 \mathrm{~K}$ in $\chi^{-1}$ (inset) signal onset of the coupled COantiferromagnetic phase transition and of weak long-range order, respectively, as described in the text; (c), X-ray photoelectron spectroscopy (XPS) analysis indicating evidence of $\mathrm{Mn}^{3+}$ and $\mathrm{Mn}^{4+}$, and no evidence of $\mathrm{Mn}^{2+}$; (d), resistivity as a function of temperature $\rho(T)$ in samples of $(\mathrm{Ba}-\mathrm{Ti}$-Mn-O) with differing $\mathrm{Ba}: \mathrm{Mn}$ : Ti ratios A: $1: 2: 5\left(\mathrm{BaMn}_{2} \mathrm{Ti}_{5} \mathrm{O}_{14.5}\right)$, B: 1:3:4 (BMT-134), and C: $1: 4: 3\left(\mathrm{BaMn}_{4} \mathrm{Ti}_{3} \mathrm{O}_{14}\right)$. The dramatic change in character of $\rho(T)$ in the case of BMT-134 provides additional evidence of the potential onset of a charge-ordering transition near $120 \mathrm{~K}$.

Temperature-dependent DC magnetic susceptibility (Fig. 2a) shows paramagnetic behavior from 120-300 K, with two distinct magnetic transitions at lower temperatures. The paramagnetic behavior fits classical Curie-Weiss behavior (Fig. 2a), $\chi(T)=C /(T-\theta)$, with $C=2.44$ and $\theta=-107 \mathrm{~K}$. The calculated effective moment per Mn cations, $4.4 \mu_{\mathrm{B}}$, further supports the presence of $\mathrm{Mn}^{3+}$ and $\mathrm{Mn}^{4+}$ cations with a ratio of $1: 1$. The compound stoichiometry is thus confirmed, consistent with all three independently verified sources: structure refinement, valence and magnetic analysis. The $\mathrm{Mn}$ sites in the lattice are all octahedral in coordination, with a splitting of the $d$-orbitals giving rise to the $e_{g} / t_{2 g}$ crystal field configuration.

We suppose that the $\mathrm{MnO}_{6}$ chains exhibit antiferromagnetic (AFM) ordering along the $c$ crystallographic direction, with weak superexchange between the $\mathrm{MnO}_{6}$ chains through the Ti-O-Ti bonds in between, as would be expected for such quasi-one-dimensional structures. As a result, the first magnetic ordering takes place below $T_{N} \approx 120 \mathrm{~K}$, where a clear deviation from the Curie-Weiss law, and an anomaly in the resistivity-versus-temperature behavior (Fig. 2c) indicate the onset of antiferromagnetism of collinear spins along the 1-D chains and, possibly, provide initial evidence of a charge-ordered state. A second magnetic transition appears at $T_{N} \sim 42 \mathrm{~K}$ (Fig. 2a). This low-temperature transition may be the result of non-collinear spin canting originating from Dzyaloshinskii-Moriya ${ }^{49}$ interactions in slightly distorted $\mathrm{Mn}-\mathrm{O}-\mathrm{Mn}$ bonds, and/or spin reorientation anticipated for a magnetically ordered, highly spin-frustrated hollandite structure ${ }^{50,51}$ As a result, a long-range weak ferromagnetic order is observed and gives rise to a hysteretic behavior of the net magnetic moment (Fig. 2b). The saturation magnetization at $1.84 \mathrm{~K}$ is $\sim 1 \mu_{\mathrm{B}}$, equal to the difference between $\mathrm{Mn}^{3+}$ and $\mathrm{Mn}^{4+}$ moments. As evident from the structural analysis of hollandites, the Mn-O-Mn bonds in the chains of $\mathrm{MnO}_{6}$ octahedra have an angle ranging from about $80^{\circ}$ to $100^{\circ}$, which could enable a superexchange toward ferromagnetic order in the $\mathrm{Mn}^{3+}-\mathrm{O}-\mathrm{Mn}^{3+}$ bond, based on the GoodenoughKanamori rule ${ }^{52,53}$. However, due to a considerable contribution from the double exchange along the $\mathrm{Mn}^{3+}-\mathrm{O}-\mathrm{Mn}^{4+}$ bonds, $\mathrm{e}_{\mathrm{g}}$ orbital ordering as well as lattice distortions, the antiferromagnetic order is frustrated ${ }^{50,51}$, and ultimately hard to predict.

In addition to the magnetic ordering described above, BMT-134 exhibits remarkably unambiguous ferroelectric switching (Fig. 3ad). Ferroelectric polarization hysteresis data were collected in mesoscopic samples (see Methods) of $30-\mu \mathrm{m}$ thick BMT-134 on heating from $77 \mathrm{~K}$ to $300 \mathrm{~K}$ under vacuum with different poling rates. Hysteresis loops with noticeable polarization saturation were observed at temperatures of up to $\sim 160 \mathrm{~K}$, above which a significant charge leakage contribution made the ferroelectric loops appear to be those of a lossy dielectric. For $\mathrm{T}<110 \mathrm{~K}$ polarization loops are weakly saturated at a poling rate as low as $5 \mathrm{~Hz}$, primarily due to both an increasingly stronger polarization at lower temperatures and to insufficiently applied bias during the measurements (100 V). In this case a long poling rate $(5 \mathrm{~Hz})$ indeed resulted in slightly saturated loops (Fig. 3a); however, decreasing the poling rate further led to an undesirable leakage contribution making the saturation indistinguishable. According to Landau theory for a typical displacive- 

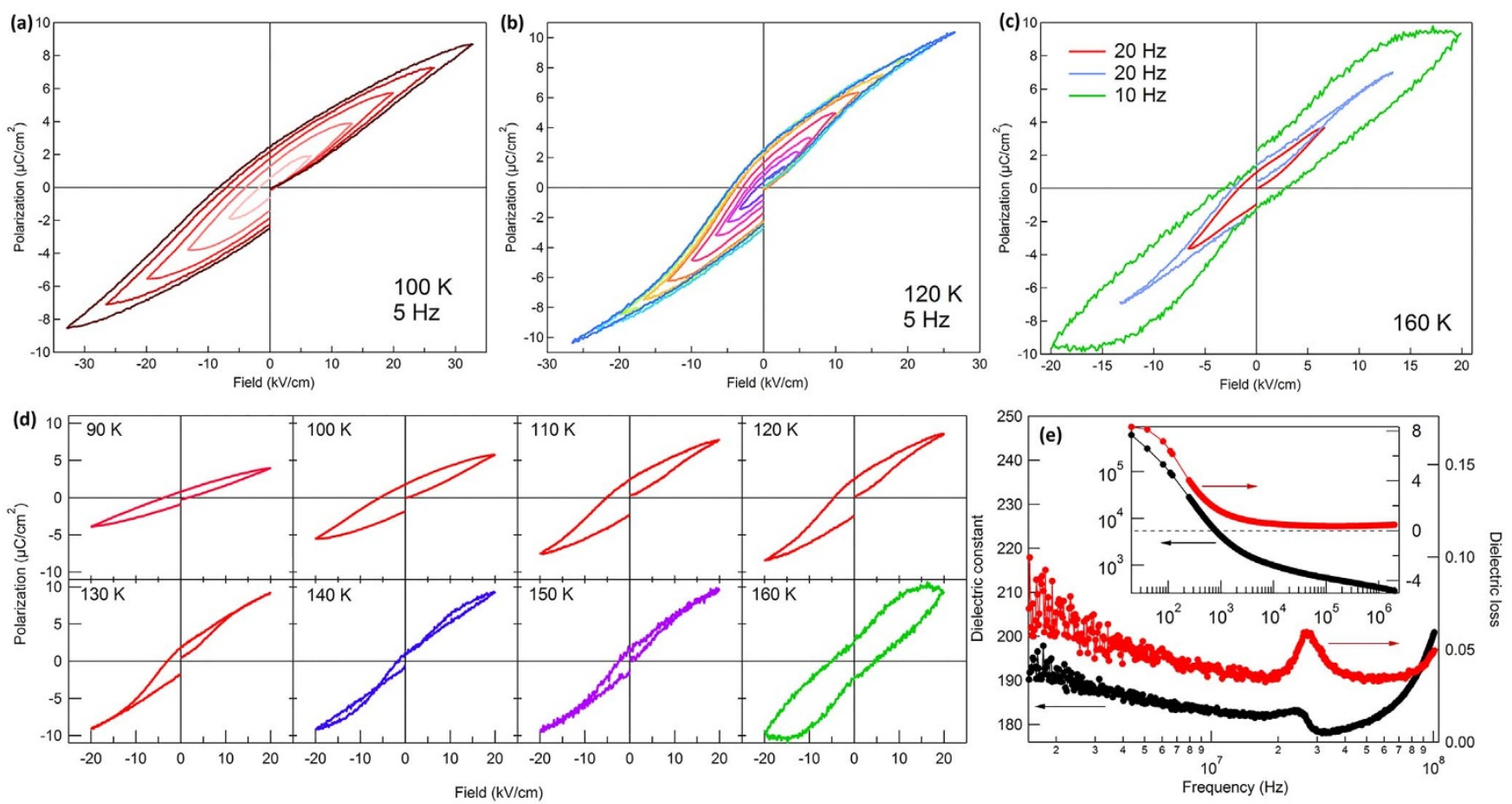

Figure 3 Ferroelectric and dielectric measurements of BMT-134. Ferroelectric hysteresis measured in BMT-134 at (a) $100 \mathrm{~K}$ and (b) $120 \mathrm{~K}$, each at a $5 \mathrm{~Hz}$ poling rate, exhibiting ferroelectric switching and saturation at $120 \mathrm{~K}$; (c) at $160 \mathrm{~K}$ unambiguous switching and saturation can be observed only at a higher poling rate due to stronger charge leakage. (d) Variation in $\mathrm{P}(\mathrm{E})$ loops in the same sample using identical voltage sweep rate of $5 \mathrm{~Hz}$, indicating an onset of leakage at this poling rate with temperature above $\sim 150 \mathrm{~K}$, below which ferroelectric switching and polarization saturation is observed. At $100 \mathrm{~K}$ and below, the value of saturation polarization for a given applied field is seen to decrease for decreasing T, presumably due to application of an insufficiently large field and incomplete poling, consistent with Landau theory for a displacive transition. (e) Frequency-dependent dielectric properties of the paraelectric phase in the ranges of $1-100 \mathrm{MHz}$ and $100 \mathrm{~Hz}-1 \mathrm{MHz}$ (inset) at $300 \mathrm{~K}$, together with dielectric loss (shown in red).

type ferroelectric, the field at which polarization saturates is expected to decrease for increasing temperature. Accordingly, ferroelectric switching with a complete saturation was observed at temperatures at and above $110 \mathrm{~K}$ (Fig. 3b) and up to $\sim 160-180 \mathrm{~K}$, the latter shown by the red- and blue-colored loops in Fig. 3c. To the best of our knowledge, there are no experimental reports of ferroelectricity for the hollandite crystal class, with one theoretical prediction, that we now confirm ${ }^{54}$. We can attribute the observation of ferroelectricity in BMT-134 to an off-center shift of $\mathrm{Ti}$ atoms inside $\mathrm{TiO}_{6}$ octahedra, in the $c$ direction, along the chain of edge-sharing octahedra, consistent with the quasi-1D character. This can be compared to other multiferroics with octahedral coordination of manganese ions (e.g. $\mathrm{BaMnO}_{3}{ }^{55}$ ), in which the neighboring $\mathrm{MnO}_{6}$ octahedra chains are also presumed to have ferroelectrically active $\mathrm{Mn}-\mathrm{O}$ covalent bonds, but structure-property measurements are complicated by cation disorder. Another possible explanation for ferroelectricity in BMT-134, that is not mutually exclusive to displacive behavior, is the onset of a $\mathrm{Mn}^{\mathrm{III}} / \mathrm{Mn}^{\mathrm{IV}}$ charge-ordered state. The presence of $\mathrm{Mn}^{3+}$ and $\mathrm{Mn}^{4+}$ with $1: 1$ molar ratio, verified independently by structural, spectroscopic and magnetic characterization, provides a substantive basis for interpretation of the sudden change in resistivity behavior, the noticeably stronger ferroelectric behavior, and the close proximity of these two events to the first antiferromagnetic transition at $\sim 110 \mathrm{~K}$. A stable charge-ordered configuration of $\mathrm{Mn}^{4+}$ and $\mathrm{Mn}^{3+}$ (Fig. 1b) is expected to be strongly bound to the spin order, supported by Hartree-Fock calculations $s^{53}$. When combined with a certain orbital order (as in $\mathrm{Pr}_{0.5} \mathrm{Ca}_{0.5} \mathrm{MnO}_{3}{ }^{56}$ ) or spin order (as in $\mathrm{TbMn}_{2} \mathrm{O}_{5}{ }^{57}$ ), or being on a geometrically frustrated lattice (as in $\mathrm{LuFe}_{2} \mathrm{O}_{4}{ }^{27}$ ), a chargeordered state was proposed to be the origin or a promoter of ferroelectric order ${ }^{28}$. BMT-134 exhibits a remarkably large dielectric response: The dielectric constant of a BMT-134 film, $\varepsilon$ is $>10^{5}$ at low frequencies, $>10^{4}$ up to $1 \mathrm{kHz},>10^{3}$ up to $1 \mathrm{MHz}$, and maintains a value of $\sim 200$ (190-200) from 1-100 MHz (DF $<0.1$, Fig. 3e). At higher frequencies $(>1 \mathrm{MHz})$ the contribution to the real part of permittivity is largely intrinsic, with no contributions from spacecharge or leakage. Again, we could attribute such a large intrinsic $\varepsilon$ to the presence of significant electron correlations that result in charge order, and/or $\mathrm{Ti}^{4+}$ - based dipolar distortion, below the critical field strength for ferroelectricity. The giant dielectric constant at low frequency range $(<1 \mathrm{kHz})$ is related to internal barrier-layer capacitance, in which the chains of edge-shared $\mathrm{TiO}_{6}$ octahedra act as barrier layers, similar to the case reported for $\mathrm{CaCu}_{3} \mathrm{Ti}_{4} \mathrm{O}_{12}$ $(\mathrm{CCTO})^{58}$. The critical difference between the CCTO and BMT134 is the presence of mixed-valence manganese ions in the latter, such that the room-temperature valence of the $\mathrm{Mn}$ ions can alternate between +3 and +4 to create a so-called charge disordered system. The long distance conductivity due to hopping $e_{g}$ electrons (double exchange) makes a decreasing contribution to the leakage with increasing impedance frequency, whereas the contribution of instantaneous dipoles to the permittivity, due to localized charge ordering, increases when the dipolar relaxation time $\left(\tau \sim 10^{-6}\right.$ $10^{-9}$ ) approaches the period of the applied electric field.

In conclusion, the attractive prospect of combining switchable and spontaneous magnetic and electrical polarization in a single-phase system is known to be limited by physical restrictions on their coexistence, as well as chemical limitations to the synthesis of novel structures and compounds. In the quest for new multifunctional materials composed of earth-abundant elements, we demonstrate the coexistence of stable long-range ferromagnetic and ferroelectric order in a hollandite crystal structure possessing a quasi-one-dimensional character, evidenced by switching hysteresis. With the introduction of this new family of non-perovskite oxide multiferroics, we aim to stimulate further inquiry into the mechanisms for the evolution of coupled ferroic orders, in this and other single-phase materi- 
als, and advocate for a wider search for compositions that retain or improve upon the degree of multi-functionality.

\section{Methods}

Synthesis of Ba-Mn-Ti oxide frameworks. The method we employ here is referred to by our group as gel-collection, due to the visible rod-like gel that forms naturally from the mother solution (see examples in Supplementary information, Figure S1). This solid gel, formed due to gentle aggregation of nanoparticles containing the correct product stoichiometry, can be readily extracted from solution and further processed, to obtain bulk or nanocrystalline products. In a typical synthesis for $\mathrm{BaMn}_{3} \mathrm{Ti}_{4} \mathrm{O}_{1425}$ (BMT-134), mmol quantities of barium isopropoxide, manganese acetylacetonate and titanium isopropoxide were mixed together in pure ethanol with the molar atomic ratio $1: 3: 4$. After forming a clear and transparent solution with magnetic stirring, the liquid was transferred to an autoclave and heated to $150^{\circ} \mathrm{C}$ for $24 \mathrm{~h}$. The gel-rod was removed from the supernatant and further sintered at $700^{\circ} \mathrm{C}$. Pure phase nanocrystal powders of $\mathrm{BaMn}_{3} \mathrm{Ti}_{4} \mathrm{O}_{14.25}$ were obtained that could be either (i) redispersed in alcohol solvents for obtaining discrete nanocrystals or spin coated thin films prepared by evaporatively driven assembly; or (ii) powder pressed and/or sintered further to obtain nanostructured monoliths. By varying precursor concentration, samples containing differing ratios of $\mathrm{Ba}: \mathrm{Mn}: \mathrm{Ti}$ are possible. This resulted in the preparation of BMT-125 $\left(\mathrm{BaMn}_{2} \mathrm{Ti}_{5} \mathrm{O}_{14.5}\right)$, and BMT-143 $\left(\mathrm{BaMn}_{4} \mathrm{Ti}_{3} \mathrm{O}_{14}\right)$, for which the molar ratio of oxygen was deduced from the estimated valence of the manganese present. Further information is provided in the Supplementary Information.

Synchrotron radiation XRD and PDF refinement. X-ray powder diffraction data of BMT-134 were collected at the X17A beamline at the National Synchrotron Light Source (NSLS) at Brookhaven National Laboratory at $100 \mathrm{~K}$ using the Rapid Acquisition mode at an X-ray energy of $67.577 \mathrm{keV}$. The diffraction intensities ware measured using a Perkin-Elmer flat-panel 2D detector. The experimental setup was calibrated using data from a Ni Standard sample using Fit2D and then integrated to $1 D$ intensities vs. $Q$ (magnitude of the scattering vector) using the SrXplanar program ${ }^{48}$. After background correction, the powder data were transformed to the $\mathrm{PDF}$ using the PDFgetX3 software ${ }^{47}$. The coherent scattering intensity from the measurement is normalized by the $Q$-dependent X-ray scattering factor $f$, to obtain the total scattering structure function $S(Q)$. Full details are provided in the Supplementary Information.

Atomic-resolution scanning transition electron microscopy, STEM. Samples of BMT-134 for STEM and electron energy loss spectroscopy (EELS) were prepared by depositing dilute nanocrystal in ethanol solutions on ultrathin carbon grids. We used JEOL ARM 200CF equipped with a cold field-emission gun and double sphericalaberration correctors at Brookhaven National Laboratory. All STEM and EELS were performed with $200 \mathrm{kV}$ electrons. The collection angles for high-angle annular-darkfield (HAADF) detectors were from 68 to $280 \mathrm{mrad}$. The energy resolution for EELS was about $0.5 \mathrm{eV}$ with $0.25 \mathrm{eV} / \mathrm{ch}$ dispersion.

XPS characterization. X-ray Photoelectron Spectroscopy analysis was carried out with the $\mathrm{ESCA}^{+}$system using a Al KR source $(1386.6 \mathrm{eV})$ The powders were mounted on non-conductive adhesive tapes on a stainless steel sample holder. The base pressure of the deposition chamber was $1 \times 10^{-9}$ Torr. The survey spectra in the range of $0-1386.6 \mathrm{eV}$ were recorded in $0.1 \mathrm{eV}$ steps for the sample. Curve fitting was performed after a Shirley background subtraction by a Lorenzian-Gaussian method. The binding energy scale of the system was calibrated using adventitious $C 1 \mathrm{~s}$ $284.8 \mathrm{eV}$ from foil samples. This process has an associated error of $\pm 0.1-0.2 \mathrm{eV}$. The spectrometer dispersion was adjusted to give a binding energy of $641.6 \mathrm{eV}$ for metallic $\mathrm{Mn} 2 \mathrm{p} 3 / 2$. The asymmetric Mn 2p3/2 main metal peak is found at around 640.40 with a $2 \mathrm{p} 3 / 2$ to $2 \mathrm{p} 1 / 2$ splitting of $11.10 \pm 0.02 \mathrm{eV}$. This compares well to a NIST database average of $641.6 \pm 1.0 \mathrm{eV}$ and $11.15 \pm 0.15 \mathrm{eV}$. The identical peak of $\mathrm{Mn} 3+$ has a spectrum at $638.6 \mathrm{eV}[\mathrm{CC}]$. The Mn4+ $(2 \mathrm{p} 3 / 2)$ spectrum of BMT-134 displays a narrow peak maximum near $640 \mathrm{eV}$, a distinct shoulder near $641.5 \mathrm{eV}$, which also indicate there is no $\mathrm{Mn} 2+$ in the oxides and a broad, pronounced shoulder between 642 and $645 \mathrm{eV}$. These features are also present in the calculated Mn4+ spectrum.

\section{Electric and magnetic properties measurements of BMT-134. Ferroelectric} measurements were performed on finely polished sintered $\mathrm{BaMn}_{3} \mathrm{Ti}_{4} \mathrm{O}_{14.25}$ pellets ( $30 \mu \mathrm{m}$ thick, both sides having Au electrodes deposited by thermal evaporation) at $77-300 \mathrm{~K}$ under $10^{-6}$ torr in a probe station (Lakeshore Desert Cryotronics TTP4) using a ferroelectric tester (LC, Radiant Technologies). Magnetic properties were measured by Magnetic Properties Measurement System (MPMS, Quantum Design, San Diego CA). Ferroelectric polarization was collected under $\sim 10^{-6}$ Torr from 77-300 K using a Lakeshore Model TTP4 probe station (Lake Shore Cryotronics, Westerville $\mathrm{OH}$ ) and a Precision LC Workstation (Radiant Technology, Albuquerque NM) equipped with a high voltage source and amplifier. Four-point DC temperature dependent conductivity were measured on $\mathrm{BaMn}_{3} \mathrm{Ti}_{4} \mathrm{O}_{14}$ tablets using a Physical Property Measurement System (PPMS, Quantum Design). Frequency dependent dielectric properties of $\mathrm{BaMn}_{3} \mathrm{Ti}_{4} \mathrm{O}_{14.25}$ tablets were measured by LCR Meter (Agilent, $4980 \mathrm{~A}$ ) and Impedance Analyzer (Agilent, E4991A).
1. Eerenstein, W., Mathur, N. D. \& Scott, J. F. Multiferroic and magnetoelectric materials. Nature 442, 759-65 (2006).

2. Cheong, S.-W. \& Mostovoy, M. Multiferroics: a magnetic twist for ferroelectricity. Nat. Mater. 6, 13-20 (2007).

3. Catalan, G. \& Scott, J. F. Physics and applications of bismuth ferrite. Adv. Mater. 21, 2463-2485 (2009).

4. Ramesh, R. \& Spaldin, N. A. Multiferroics: progress and prospects in thin films. Nat. Mater. 6, 21-9 (2007).

5. Lee, J. H. et al. A strong ferroelectric ferromagnet created by means of spin-lattice coupling. Nature 466, 954-8 (2010).

6. Wu, S. M. et al. Reversible electric control of exchange bias in a multiferroic fieldeffect device. Nat. Mater. 9, 756-761 (2010).

7. Mannhart, J., Blank, D. H. A., Hwang, H. Y., Millis, A. J. \& Triscone, J.-M. Twodimensional electron gases at oxide interfaces. MRS Bull. 33, 1027-1034 (2008).

8. Homes, C. C., Vogt, T., Shapiro, S. M., Wakimoto, S. \& Ramirez, A. P. Optical response of high-dielectric-constant perovskite-related oxide. Science 293, 673-6 (2001).

9. Zhu, Y. et al. Nanoscale disorder in $\mathrm{CaCu}_{3} \mathrm{Ti}_{4} \mathrm{O}_{12}$ : a new route to the enhanced dielectric response. Phys. Rev. Lett. 99, 037602 (2007).

10. Zhang, X. X. et al. Magnetocaloric effect in $\mathrm{La}_{0.67} \mathrm{Ca}_{0.33} \mathrm{MnO}_{\delta}$ and $\mathrm{La}_{0.60} \mathrm{Y}_{0.07} \mathrm{Ca}_{0.33} \mathrm{MnO}_{\delta}$ bulk materials. Appl. Phys. Lett. 69, 3596 (1996).

11. Mischenko, A. S., Zhang, Q., Scott, J. F., Whatmore, R. W. \& Mathur, N. D. Giant electrocaloric effect in thin-film $\mathrm{PbZr}_{0.95} \mathrm{Ti}_{0.05} \mathrm{O}_{3}$. Science 311, 1270-1 (2006).

12. Cheong, S.-W. Transition metal oxides: the exciting world of orbitals. Nat. Mater. 6, 927-928 (2007).

13. Kitagawa, Y. et al. Low-field magnetoelectric effect at room temperature. Nat. Mater. 9, 797-802 (2010).

14. Grinberg, I. et al. Perovskite oxides for visible-light-absorbing ferroelectric and photovoltaic materials. Nature 503, 509-12 (2013).

15. Scott, J. F. Room-temperature multiferroic magnetoelectrics. NPG Asia Mater. 5, e72 (2013).

16. Hill, N. A. Why are there so few magnetic ferroelectrics? J. Phys. Chem. B 104, 6694-6709 (2000).

17. Khomskii, D. Classifying multiferroics: mechanisms and effects. Physics (College. Park. Md). 2, 20 (2009)

18. Akbashev, A. R. \& Kaul, A. R. Structural and chemical aspects of the design of multiferroic materials. Russ. Chem. Rev. 80, 1159-1177 (2011).

19. Bibes, M. Towards a magnetoelectric memory. Nat. Mater. 7, 7-9 (2008).

20. Béa, H., Gajek, M., Bibes, M. \& Barthélémy, A. Spintronics with multiferroics. J. Phys. Condens. Matter 20, 434221 (2008).

21. Scherwitzl, R. et al. Metal-insulator transition in ultrathin $\mathrm{LaNiO}_{3}$ films. Phys. Rev. Lett. 106, 246403 (2011).

22. Srinivasan, G. Magnetoelectric composites. Annu. Rev. Mater. Res. 40, 153-178 (2010).

23. Liu, X. et al. Magnetoelectricity in $\mathrm{CoFe}_{2} \mathrm{O}_{4}$ nanocrystal-P(VDF-HFP) thin films. Nanoscale Res. Lett. 8, 374 (2013).

24. Vaz, C. A. F., Hoffman, J., Ahn, C. H. \& Ramesh, R. Magnetoelectric coupling effects in multiferroic complex oxide composite structures. Adv. Mater. 22, 2900-18 (2010).

25. Ma, J., Hu, J., Li, Z. \& Nan, C.-W. Recent progress in multiferroic magnetoelectric composites: from bulk to thin films. Adv. Mater. 23, 1062-87 (2011).

26. Yu, P., Chu, Y. H. \& Ramesh, R. Emergent phenomena at multiferroic heterointerfaces. Philos. Trans. A. Math. Phys. Eng. Sci. 370, 4856-71 (2012).

27. Ikeda, N. et al. Ferroelectricity from iron valence ordering in the charge-frustrated system LuFe2O4. Nature 436, 1136-8 (2005).

28. Van den Brink, J. \& Khomskii, D. I. Multiferroicity due to charge ordering. J. Phys. Condens. Matter 20, 434217 (2008).

29. Angst, M. Ferroelectricity from iron valence ordering in rare earth ferrites? Phys. Status Solidi 1-18 (2013).

30. Kimura, T., Sekio, Y., Nakamura, H., Siegrist, T. \& Ramirez, A. P. Cupric oxide as an induced-multiferroic with high-TC. Nat. Mater. 7, 291-4 (2008).

31. Babkevich, P. et al. Electric field control of chiral magnetic domains in the hightemperature multiferroic CuO. Phys. Rev. B 85, 134428 (2012).

32. Sanchez, D. a. et al. Room-temperature single phase multiferroic magnetoelectrics: $\mathrm{Pb}(\mathrm{Fe}, \mathrm{M})_{\mathrm{x}}(\mathrm{Zr}, \mathrm{Ti})_{(1-\mathrm{x})} \mathrm{O}_{3}[\mathrm{M}=\mathrm{Ta}, \mathrm{Nb}]$. J. Appl. Phys. 113, 074105 (2013).

33. Evans, D. M. et al. Magnetic switching of ferroelectric domains at room temperature in multiferroic PZTFT. Nat. Commun. 4, 1534 (2013).

34. Keeney, L. et al. Magnetic field-induced ferroelectric switching in multiferroic Aurivillius phase thin films at room temperature. J. Am. Ceram. Soc. 96, 2339-2357 (2013).

35. Keeney, L. et al. Room temperature ferroelectric and magnetic investigations and detailed phase analysis of Aurivillius phase $\mathrm{Bi}_{5} \mathrm{Ti}_{3} \mathrm{Fe}_{0.7} \mathrm{Co}_{0.3} \mathrm{O}_{15}$ thin films. J. Appl. Phys. 112, 052010 (2012).

36. Keith, G. M. et al. Synthesis, srystal structure, and characterization of $\mathrm{Ba}\left(\mathrm{Ti}_{0.5} \mathrm{Mn}_{0.5}\right) \mathrm{O}_{3}$ : a high permittivity $12 \mathrm{R}$-type hexagonal perovskite. Chem Mater 16, 2007-2015 (2004).

37. Miranda, L. et al. Polytypism in the $\mathrm{BaMn}_{0.85} \mathrm{Ti}_{0.15} \mathrm{O}_{3-\delta}$ system $(0.07 \leq \delta \leq 0.34)$. Structural, magnetic, and electrical characterization of the 9R-polymorph. Chem. Mater. 22, 4320-4327 (2010)

38. Prieto, A. L., Siegrist, T. \& Schneemeyer, L. F. New barium manganese titanates prepared under reducing conditions. Solid State Sci. 4, 323-327 (2002). 
39. Melbourne, P. et al. Preparation and structure refinement of synthetic Ti3+ containing Lindsleyite, $\mathrm{BaMn}_{3} \mathrm{Ti}_{18} \mathrm{O}_{38}$. Can. Mineral. 33, 1083-1089 (1995).

40. Foley, J. A., Hughes, J. M. \& Drexler, J. W. Redledgite,

$\mathrm{Ba}_{\mathrm{x}}\left([\mathrm{Cr}, \mathrm{Fe}, \mathrm{V}]^{3+}{ }_{2 \mathrm{x}} \mathrm{Ti}_{(8-2 \mathrm{x})} \mathrm{O}_{16}\right.$, The I4/m structure and elucitation of the sequence of tunnel Ba cations. Can. Mineral. 35, 1531-1534 (1997).

41. Miura, H. The crystal structure of hollandite. Mineral. J. 13, 119-129 (1986)

42. Yang, P., Zhao, D., Margolese, D. I., Chmelka, B. F. \& Stucky, G. D. Generalized syntheses of large-pore mesoporous metal oxides with semicrystalline frameworks. Nature 396, 152-155 (1998).

43. Schwartz, R. W. Chemical solution deposition of perovskite thin films. Chem. Mater. 4756, 2325-2340 (1997).

44. Niederberger, M. Nonaqueous sol-gel routes to metal oxide nanoparticles. Acc. Chem. Res. 40, 793-800 (2007)

45. O’Brien, S., Brus, L. \& Murray, C. B. Synthesis of monodisperse nanoparticles of barium titanate: toward a generalized strategy of oxide nanoparticle synthesis. J. Am. Chem. Soc. 123, 12085-12086 (2001).

46. Egami, T. \& Billinge, S. J. L. Underneath the Bragg peaks, Volume 16: Structural analysis of complex materials (Pergamon Materials Series) 2nd Edition. (Pergamon Press, 2013).

47. Juhás, P., Davis, T., Farrow, C. L. \& Billinge, S. J. L. PDFgetX3: a rapid and highly automatable program for processing powder diffraction data into total scattering pair distribution functions. J. Appl. Crystallogr. 46, 560-566 (2013).

48. Yang, X., Juhas, P. \& Billinge, S. J. L. Estimating statistical uncertainties on powder diffraction and small angle scattering data from 2-D planar $\mathrm{x}$-ray detectors. (2014).

49. Dzyaloshinskii, I. Theory of helical structures in antiferromagnets I: Nonmetals. Sov. Phys. JETP 19, 960-971 (1964)

50. Ishiwata, S., Bos, J. W. G., Huang, Q. \& Cava, R. J. Structure and magnetic properties of hollandite $\mathrm{Ba}_{1.2} \mathrm{Mn}_{8} \mathrm{O}_{16}$. J. Phys. Condens. Matter 18, 3745-3752 (2006).

51. Crespo, Y., Andreanov, a. \& Seriani, N. Competing antiferromagnetic and spinglass phases in a hollandite structure. Phys. Rev. B 88, 014202 (2013).

52. Tian, G.-S. Antiferromagnetic correlation in the half-filled strongly correlated electron models at nonzero temperature: A rigorous result. Phys. Rev. B 63 , 224413 (2001).

53. Fukuzawa, M., Otsuki, D. O. \& Mizokawa, T. Spin - charge - orbital ordering in Hollandite-type manganites studied by model Hartree - Fock calculation. J. Phys. Soc. Japan 82, 074708-1-5 (2013).

54. Abrahams, S. C. Systematic prediction of new inorganic ferroelectrics in point group 4. Acta Crystallogr. B55, 494-506 (1999).

55. Rondinelli, J., Eidelson, A. \& Spaldin, N. Non-d0 Mn-driven ferroelectricity in antiferromagnetic $\mathrm{BaMnO}_{3}$. Phys. Rev. B 79, 205119 (2009).

56. Brinkman, A. et al. Magnetic effects at the interface between non-magnetic oxides. Nat. Mater. 6, 493-6 (2007).

57. Hur, N., Park, S., Sharma, P. \& Ahn, J. Electric polarization reversal and memory in a multiferroic material induced by magnetic fields. Nature 429, 392-395 (2004).

58. Adams, T. B., Sinclair, D. C. \& West, A. R. Giant barrier layer capacitance effects in $\mathrm{CaCu}_{3} \mathrm{Ti}_{4} \mathrm{O}_{12}$ ceramics. Adv. Mater. 14, 1321-1323 (2002).

\section{Acknowledgments}

This work was supported by the Advanced Research Project Agency for Energy (ARPA-e), ADEPT DE-AR0000114, and the National Science Foundation under award NSF IDR \#1014777. Support is also acknowledged from NSF MIRT \#1122594 and the Center for Exploitation of Nanostructures in Sensors and Energy Systems under NSF Cooperative Agreement \#0833180. Microscopy work at BNL and use of the National Synchrotron Light Source, Brookhaven National Laboratory was supported by the U.S. Department of Energy, Office of Science, Office of Basic Energy Sciences (U.S. DOE-BES), under Contract No. DE-AC02-98CH10886. X-ray analysis at Columbia University was supported as part of the Energy Frontier Research Center (EFRC) funded by the U.S. DOE-BES under Award Number DE-SC0001085. J.E.S. acknowledges support from the U.S. Army Research Office (W911NF-08-1-0067) and the NSF/SRC-sponsored Nanoelectronics in 2020 and Beyond Program (DMR 1124696).

\section{Author contributions}

S.L. and S.O'B. devised the research strategy and designed the experiments. L.H. advised on chemical synthesis strategy. J.L. advised on multiferroic materials and measurements. X.Y. and S.B. performed the X-ray PDF experiments (at Brookhaven National Laboratories), simulation/refinement and prepared the PDF data presented in Figurela. X.L. and MG.H. (Y. Zhu advising) performed the TEM, STEM and analytical experiments at Brookhaven National Laboratories, data presented in Figure 1b, 1c and 1d. W.L. performed the XPS data collection and analysis, presented in Figure 2c. X.L. and A.C. assisted with TGA/DSC and AFM characterization. L.Z. and L.K.-E. assisted S.L. with the magnetic/conductivity characterization and interpretation, presented in Figure 2a, $2 \mathrm{~b}$ and 2d. A.R.A., S.L. and J.S performed the ferroelectric and dielectric measurements presented in Figure 3. S.L. and S.O'B. wrote the original manuscript; A.R.A. and J.S. contributed extensively to the final manuscript, especially the multiferroic properties intepretation. S.B., J.L., Y.Z., M.G.H. and L.K.-E. all commented on the manuscript during preparation.

\section{Additional information}

Supplementary information accompanies this paper at http://www.nature.com/ scientificreports

Competing financial interests: The authors declare no competing financial interests.

How to cite this article: Liu, S. et al. Hollandites as a new class of multiferroics. Sci. Rep. 4, 6203; DOI:10.1038/srep06203 (2014).

This work is licensed under a Creative Commons Attribution-NonCommercialNoDerivs 4.0 International License. The images or other third party material in this article are included in the article's Creative Commons license, unless indicated otherwise in the credit line; if the material is not included under the Creative Commons license, users will need to obtain permission from the license holder in order to reproduce the material. To view a copy of this license, visit http:// creativecommons.org/licenses/by-nc-nd/4.0/ 